\title{
Interdisziplinäre Therapiekonzepte bei kolorektalen Lebermetastasen
}

\author{
Gesprächsleiter: Peter Neuhaus (Berlin) \\ Teilnehmer: $\quad$ Claus-Henning Köhne (Oldenburg) Hauke Lang (Mainz) \\ Ulf P. Neumann (Aachen) Hans J. Schlitt (Regensburg) \\ Henning Schulze-Bergkamen (Heidelberg)
}

Frage 1: Welche Untersuchungen sollen bei potenziell operablen Lebermetastasen unbedingt gemacht werden? Welche sollen bei inoperablen Lebermetastasen durchgeführt werden und mit welchem Ziel? Wie sieht lhre persönliche Vorgehensweise aus?

Köhne: Bei Patienten mit potenziell operablen Lebermetastasen sollte eine Computertomographie (CT) des Thorax und eine Kernspinuntersuchung der Leber erfolgen. Letztere stellt mit hoher Sensitivität das Ausmaß des intrahepatischen Befalls fest, insbesondere wenn mit leberspezifischem Kontrastmittel (KM) untersucht wird. Die CT-Thorax-Untersuchung führt zum Ausschluss von pulmonalen Metastasen. Der Stellenwert einer Positronenemissionstomographie(PET)CT-Untersuchung wird zurzeit noch kontrovers diskutiert, allerdings können nach einzelnen Berichten bei etwa einem Viertel der Patienten extrahepatische Metastasen gefunden werden, die dann zu einer Änderung des Therapiekonzepts führen können.

Aufgrund des verbesserten perioperativen Managements und der relativ niedrigen Mortalität und Morbidität einer Leberoperation diskutieren wir jedoch zunehmend auch den Stellenwert einer palliativen Leberoperation, womit die Frage des Nachweises kleinerer pulmonaler Metastasen bzw. eines geringeren extrahepatischen Befalls in den Hintergrund treten könnte.

Nach dem eigenen Vorgehen führen wir bei allen Patienten eine CT-Thorax-Untersuchung sowie eine Kernspinuntersuchung des Abdomens durch. Üblicherweise ist bereits auswärts eine CT-Untersuchung des Abdomens veranlasst worden.

Lang: Die Bildgebung kolorektaler Lebermetastasen bei Erstdiagnose und beim Re-Staging nach Chemotherapie hat das Ziel, die potenzielle Resektabilität zu definieren und eine mögliche Leberresektion zu planen. Zudem sollten extrahepatische Metastasen ausgeschlossen werden. Standarddiagnostik ist die KM-verstärkte Spiral-CT. Sie soll das Resektionsausmaß und das verbleibende Restvolumen der Leber definieren. In schwierigen Fällen kann hierzu eine computergestützte Volumetrie durchgeführt werden. Artdiagnostisch unklare Herdbefunde können durch die Sonographie und die Kernspintomographie im Einzellfall weiter eingegrenzt werden. Bestehen Unklarheiten bezüglich der technischen Resektabilität, kann eine dreidimensionale Rekonstruktion bei ausgewählten Fällen die Operationsplanung erleichtern.

Bei irresektablen Lebermetastasen hängt die Diagnostik vom Ziel der Untersuchung ab. Sogenannte «Targetläsionen» zur Therapiekontrolle einer palliativen Chemotherapie können meist sonographisch überwacht werden. Eine CT sollte dann durchgeführt werden, wenn im Rahmen eines ReStagings die Möglichkeit einer sekundären Resektabilität evaluiert werden soll.

In der eigenen Klinik bilden die Sonographie und die CT die Standarddiagnostik zur Operationsplanung bei kolorektalen Lebermetastasen. In einzelnen Fällen wird die Diagnostik durch 3-D-Rekonstruktionen ergänzt. Eine eigenhändig durchgeführte präoperative Sonographie ermöglicht dem erfahrenen Untersucher und Operateur häufig eine hilfreiche persönliche Einschätzung der Situation.

Neumann: Potenziell operable Lebermetastasen: Die kontrastverstärkte Mehrschicht-CT hat sich als die präoperative Standard-Bildgebung durchgesetzt. Die Magnetresonanztomographie (MRT) kann bei unklaren Leberläsionen und zur genaueren Abklärung der lokalen Verhältnisse vor einer Leberteilresektion wichtige Zusatzinformationen liefern. Der

\section{KARGER}

Fax +497614520714

Information@Karger.de

www.karger.com (c) 2012 S. Karger GmbH, Freiburg

Accessible online at:

www.karger.com/vim 
Einsatz spezieller Pulssequenzen wie die diffusionsgewichtete Bildgebung oder die Verwendung von leberspezifischen KM erlaubt eine optimale Detektion auch kleinster Läsionen. Die intraoperative Sonographie (IOUS) erhöht die diagnostische Sensitivität und ist meines Erachtens eine wichtige Voraussetzung für die erfolgreiche Lebermetastasenchirurgie. Bei Verdacht auf extrahepatische Befunde bzw. okkulte entfernte Lymphknotenmetastasen ergibt sich die Indikation zur PETCT zur Ganzkörperdiagnostik. Präoperativ sollte neben der Schnittbildgebung zur Darstellung der Tumorlast in der Leber sichergestellt werden, dass kein Lokalrezidiv des Primarius vorliegt. Insbesondere um die lokale Resektabilität zu klären, präferieren wir eine MRT sowie ein CT-Thorax. Vor ausgedehnten Resektionen sind präoperativ eine Volumetrie der verbleibenden Restleber sowie eine Leberfunktionsbeurteilung notwendig.

Inoperable Lebermetastasen: Für die Beurteilung des Therapieerfolgs einer Systemtherapie bei Lebermetastasen mithilfe der radiologischen RECIST-Kriterien sollte eine CT ausreichend sein und erlaubt hierbei auch eine Re-Evaluation hinsichtlich der potenziellen sekundären Resektabilität.

Schlitt: Bei potenziell operablen Lebermetastasen - und damit potenziell kurativem Therapieansatz - muss durch ausführliche präoperative Bildgebung geklärt werden:

1. Die möglichst genaue Zahl und Lokalisation der intrahepatischen Metastasen, um die Resektabilität optimal abschätzen und die Operationsstrategie planen zu können. Hierzu ist in der Regel ein KM-CT des Abdomens mit arterieller, portalvenöser und venöser KM-Phase der Leber eine gute Grundlage. Insbesondere nach chemotherapeutischer Vorbehandlung sind jedoch die Metastasen im CT oft schlecht zu erkennen, sodass hier alternativ oder additiv eine MRT-Untersuchung der Leber erfolgen muss, idealerweise mit leberspezifischem KM. Darüber hinaus kann eine KM-Sonographie zusätzliche Informationen liefern.

2. Ob Hinweise auf extrahepatische Tumormanifestation bestehen, insbesondere Lymphknotenmetastasen, Lungenmetastasen oder ein Lokalrezidiv. Hierzu ist zusätzlich zum KM-CT des Abdomens ein Thorax-CT essenziell. Zusätzlich sollte - insbesondere in der Rezidivsituation - ein ${ }^{18}$ F-Fluorodeoxyglukose(FDG)-PET-CT durchgeführt werden. Weitere Untersuchungen (z.B. Schädel-CT oder Knochenszintigraphie usw.) erscheinen nur bei klinischem Verdacht notwendig zu sein.

Zur weiteren Verlaufskontrolle sollten präoperativ außerdem die Tumormarker CEA und CA 19-9 sowie der CRP-Wert bestimmt werden. Intraoperativ sollte zum Ausschluss weiterer Lebermetastasen in jedem Fall eine IOUS der Leber erfolgen.

Bei definitiv irresektablen Lebermetastasen wird in der Regel ein KM-CT von Thorax und Abdomen sowie eine Bestimmung der Tumormarker als Basis für eine palliative Therapie und zur Verlaufsbeurteilung sinnvoll und ausreichend sein.
Schulze-Bergkamen: Bei potenziell operablen Lebermetastasen steht zuerst die Frage der extrahepatischen Tumormanifestationen im Vordergrund:

1. Ist der Primärtumor resektabel? Ist der Primärtumor lymphatisch metastasiert?

2. Liegen sonstige lymphatische Fernmetastasen oder pulmonale Metastasen vor?

3. Gibt es Hinweise für ossäre oder zerebrale Metastasen?

Rationale: Je nach extrahepatischen Manifestationen der Tumorerkrankung kann sich eine palliative Therapiesituation ergeben, in der eine Leberoperation dann onkologisch nicht mehr sinnvoll ist. Zudem ergeben sich aus den extrahepatischen Manifestationen entscheidende Kriterien für die Prognose; dies sollte dann in die Patientenaufklärung vor einem operativen Eingriff mit einfließen.

Die genannten Fragen sollten mittels CT-Abdomen und CT-Thorax beantwortet werden (Stellungnahme Radiologe zur Mindestanforderung an CT-Technik erforderlich). Gegebenenfalls ist ein MRT von Leber/Abdomen zu ergänzen (Stellungnahme Radiologe, siehe oben). Beim Rektumkarzinom sind eine MRT des Beckens (Stellungnahme Radiologie, siehe oben) und eine Endosonographie indiziert. Eine Tumormarkerbestimmung (CEA) trägt ebenfalls zur Prognoseabschätzung bei und sollte durchgeführt werden. Zum Ausschluss eines Zweittumors sollte eine komplette Koloskopie erfolgen.

Bei CT-morphologisch auffälligen ossären Veränderungen oder bei entsprechender Symptomatik sollte zum Ausschluss einer ossären Metastasierung ein Knochenszintigramm erfolgen.

Bei unklaren CT- bzw. MRT-Befunden hinsichtlich lymphatischer Metastasierung kann ein PET-CT erwogen werden.

Bei irresektablen (nicht potenziell resektablen) Lebermetastasen sollte vor Einleitung einer systemischen Therapie ein Ausgangsstaging erfolgen. Dazu gehören eine Schnittbildgebung (primär Oberbauchsonogramm), ein Röntgen-Thorax und eine Tumormarkerbestimmung (CEA), gegebenenfalls auch eine erweiterte Schnittbildgebung bei unklaren Befunden. Eine Knochenszintigraphie sollte vor allem bei ossärer Metastasierung erfolgen.

\section{Frage 2: Metachrone, operable Lebermetastasen werden nach der EORTC-Studie (Nordlinger) praktisch alle zunächst chemotherapiert, wenn die Patienten von Onkologen betreut werden. Bitte beziehen Sie dazu Stellung und begründen Sie Ihre Position.}

Köhne: In der EORTC-Studie [1] ergab sich insbesondere in den ersten Wochen eine deutliche Veränderung in den Kurven zum progressionsfreien Überleben (PFS). Alle Patienten galten ja primär als resektabel. Patienten, die dann intraoperativ als nicht resektabel erschienen, wurden als Behandlungsversager eingestuft, was zu einem starken Abfall der Kaplan- 
Meier-Kurve führte. Bei Patienten, die präoperativ vorbehandelt wurden, ergab sich intraoperativ in geringerem Ausmaß eine Irresektabilität. Die Überlebenskurven starten daher auf einem anderen Niveau bei etwa 78 vs. $85 \%$ und verlaufen dann annähernd parallel. Dies legt nahe, dass möglicherweise insbesondere Patienten mit grenzwertig resektablen Lebermetastasen von einer präoperativen Therapie profitieren. Dies ist einer der Gründe, warum es einige kritische Stimmen gibt, die den generellen Einsatz einer perioperativen chemotherapeutischen Behandlung nicht befürworten. Nach den aktuellen ESMO-Guidelines gilt die perioperative Chemotherapie als die Therapie der Wahl, von der bei singulären kleinen Lebermetastasen, z.B. unter $2 \mathrm{~cm}$, abgewichen werden kann. Ich persönlich betrachte Patienten mit Lebermetastasen weniger in Kategorien denn als Kontinuum der Präsentation. Bei kleineren Metastasen erscheint mir durchaus eine primäre Resektion gerechtfertigt, die dann gegebenenfalls von einer adjuvanten Therapie gefolgt werden kann. Bei Patienten, die nach einem Risiko-Score eine hohe Rückfallrate haben, erscheint mir trotz technisch möglicher Resektion zunächst eine chemotherapeutische Vorbehandlung gerechtfertigt.

Lang: Die EORTC-Studie [1] ist ohne Zweifel die methodisch hochwertigste publizierte Studie zur Frage der perioperativen Therapie primär resektabler Lebermetastasen. Allerdings sind die Ergebnisse der Studie umstritten, da in der Intentionto-treat-Analyse kein signifikanter Vorteil für eine perioperative Therapie gefunden wurde. Allerdings findet man in relevanten Subgruppen-Analysen signifikante Vorteile bezüglich des PFS durch eine perioperative Therapie. Daten für das Gesamtüberleben (OS) des Studienkollektivs wurden bislang nicht publiziert. Zudem wurden die Ergebnisse der EORTCStudie bislang nicht durch weitere unabhängige Daten bestätigt. Zusammenfassend profitiert wahrscheinlich eine Subgrupppe von Patienten mit kolorektalen Lebermetastasen von einer perioperativen Therapie. Zukünftige Analysen werden diese Patientensubgruppe eventuell besser definieren. Ein Vorteil für eine alleinige präoperative (neoadjuvante) Chemotherapie primär resektabler Metastasen ist mit der EORTC-Studie nicht untermauert.

Aus den genannten Gründen ergibt sich aus unserer Sicht (noch) kein zwingender Grund zur perioperativen Therapie primär resektabler kolorektaler Lebermetastasen. Handelt es sich um primär nicht sicher resektable Metastasen, sollte die Indikation zur präoperativen Chemotherapie großzügig gestellt werden, da durch diese ein signifikantes Downsizing der Metastasen erfolgen kann.

Neumann: In der EORTC-Studie [1] wurden Patienten, die eine perioperative Therapie erhielten, mit dem Beobachtungsarm verglichen und zeigten für das krankheitsfreie Überleben (DFS) nach 3 Jahren für alle operierten Patienten eine signifikante Verbesserung des tumorfreien Überlebens. Betrachtet man die adjuvanten Studien, so zeigen sich hier trotz veralteter Chemotherapieprotokolle absolut sehr ähnliche Ergebnisse. Zusätzlich sind die Daten für das OS der Nordlinger-Studie [1] nicht publiziert. Größere nichtrandomisierte Analysen zeigten, dass der postoperative Teil der perioperativen Therapie möglicherweise der entscheidende ist [2]. In einer 2009 publizierten retrospektiven Untersuchung ergab sich für die postoperative Chemotherapie im Vergleich zur perioperativen sogar ein verbessertes DFS und OS [3]. Insbesondere Patienten mit einer singulären metachronen Lebermetastase scheinen nicht von einer neoadjuvanten Chemotherapie zu profitieren [4]. Unter Berücksichtigung der möglicherweise häufiger auftretenden perioperativen Komplikationen und Toxizitäten muss die präoperative Chemotherapie also nach wie vor kontrovers diskutiert werden [5-8]. Aus meiner Sicht ist das Problem aller randomisierten Studien zur Lebermetastasenchirurgie kolorektaler Karzinome die Wahl des Patientenkollektivs mit zu geringer Tumorlast (z.B. Nordlinger: im Median 1 Tumor von ca. 4 cm Größe).

Die perioperative Therapie hat meines Erachtens einen wichtigen Stellenwert in der Behandlung marginal resektabler Metastasen. Derzeit wird in einer aktuellen Multicenterstudie die Sicherheit und Wirksamkeit einer perioperativen versus der postoperativen Chemotherapie mit jeweils FOLFOX plus Cetuximab geprüft (PANTER-Studie, bei K-RAS-Wildtyp). Vor allem bei älteren Patienten mit limitierter Tumorlast sehe ich für eine (peri- oder postoperative) Chemotherapie aufgrund der Nebenwirkungen keinen Vorteil.

Schlitt: Theoretisch erscheint im Stadium IV des kolorektalen Karzinoms (also immer bei Vorliegen von kolorektalen Lebermetastasen) eine systemische Chemotherapie ergänzend $\mathrm{zu}$ einer kurativen Resektion durchaus sinnvoll - und ist vermutlich im neoadjuvanten Setting konsequenter und einfacher zu verabreichen als adjuvant. Allerdings gibt es für den onkologischen «Erfolg» einer grundsätzlich durchgeführten additiven Chemotherapie - sei es vor oder nach einer R0Resektion von Lebermetastasen - bisher keine klare Evidenz. Auch die EORTC-Studie [1] konnte hierfür keine wirklich überzeugenden Daten liefern - insbesondere nicht in der Intention-to-treat-Analyse. Aus diesem Grund wird im eigenen Vorgehen bei gut resektablen kolorektalen Lebermetastasen in der Regel keine Chemotherapie vor der Resektion metachroner kolorektaler Lebermetastasen durchführt. Bei multiplen bzw. grenzwertig resektablen Metastasen wird dies im Einzelfall erwogen - insbesondere auch, um ein Ansprechen auf Chemotherapie gegebenenfalls als biologisches Selektionskriterium zu verwenden. Bei der Durchführung einer neoadjuvanten Therapie sind jedoch immer auch die möglichen Risiken bezüglich einer Leberschädigung durch die Therapie abzuwägen. Insbesondere bei längerfristiger Chemotherapie steigt das Risiko von intra- und postoperativen Komplikationen bei der Leberesektion deutlich an. Außerdem kann die funktionelle Reservekapazität der Leber durch die Vorbehandlung unter Umständen deutlich verschlechtert werden. 
Die Entscheidung für oder gegen eine neoadjuvante Therapie bei sicher oder potenziell resektablen Lebermetastasen sollte daher immer gemeinsam in einem entsprechend erfahrenen Tumorboard (d.h. in Anwesenheit eines erfahrenen Leberchirurgen!) erfolgen. Zur Klärung der Frage, ob und für welche Patienten in dieser Konstellation grundsätzlich eine neoadjuvante Therapie sinnvoll ist, müssen weitere klinische Studien durchgeführt werden, wie z.B. die aktuell angelaufenen bzw. anlaufenden Studien PANTER und PERIMAX.

Schulze-Bergkamen: Rationale/Vorteile der initialen Chemotherapie: Frühe Eradikation möglicher disseminierter Tumorzellen; wichtige Erkenntnisse hinsichtlich Tumorbiologie präoperativ (z.B. Identifikation von Patienten mit schnellem extrahepatischem Tumorprogress unter laufender systemischer Therapie, bei denen eine Leberoperation dann auch in der Regel ohne Benefit ist); die histologische Aufarbeitung kann Hinweise geben, ob eine adjuvante Chemotherapie sinnvoll ist (Regressionsgrad); höhere Wahrscheinlichkeit für R0-Resektion.

Nachteile: Hepatotoxizität der präoperativen Therapie mit Erhöhung der perioperativen Morbidität; erschwertes Auffinden von Metastasen bei kompletter Remission.

In unserem Zentrum wird bei metachroner Metastasierung die perioperative Chemotherapie aufgrund der oben genannten Vorteile favorisiert.

Frage 3: Sollten Patienten mit ungünstiger Prognose (Lokalrezidiv, Lungenmetastasen, positive Hiluslymphknoten usw.) leberreseziert werden oder gibt es prinzipiell Situationen, in denen eine Operation nicht empfehlenswert ist, obwohl eine R0-Resektion möglich wäre?

Köhne: Trotz prinzipiell möglicher R0-Resektion ist bei einigen Patienten ein solches Vorgehen onkologisch nicht gerechtfertigt, wenn z.B. Risikofaktoren eine hohe Rückfallrate anzeigen und damit eine schlechte Tumorbiologie andeuten. In diesem Fall würde ich die Patienten präoperativ vorbehandeln, um für die Patienten auch durch eine Tumorkontrolle ein besseres Zeitfenster für ein operatives Vorgehen zu schaffen und ein besseres operatives Ergebnis zu ermöglichen.

Wenn die Haupttumorlast in der Leber ist, halte ich es ebenfalls für gerechtfertigt, bei kleineren Lungenmetastasen oder Hiluslymphknoten eine Leberresektion durchzuführen. Hier wird die Haupttumorlast entfernt, wovon die Patienten höchstwahrscheinlich insgesamt profitieren werden.

Lang: Prinzipiell stellt die Leberresektion nur einen Teil der notwendigen Therapie bei einer disseminierten Tumorerkrankung dar. Eine Leberresektion sollte aber immer dann angestrebt werden, wenn bei einem Patienten eine R0-Situation, inklusive Resektion etwaiger extrahepatischer Tumormanifestation, möglich erscheint. Dabei ist jedoch jeweils die zu erwartende Morbidität des Eingriffs zu berücksichtigen. Je ungünstiger die Gesamtprognose des Patienten erscheint, desto unkomplizierter muss der Lebereingriff sein, damit der Patient zügig postoperativ z.B. einer Chemotherapie zugeführt werden kann. Dies trifft z.B. auf Patienten mit tumorbefallenen Hiluslymphknoten oder extrahepatischer Tumormanifestation zu.

Scheint ein Patient primär durch einen hepatischen Tumorprogress vital bedroht, z.B. im Falle synchron metastasierter, aber asymptomatischer kolorektaler Primärkarzinome, ist aus unserer Sicht ein Liver-first-Konzept gerechtfertigt. Die Lebermetastasierung kann auch in Situationen mit einer synchronen Lungenmetastasierung prognoselimitierend wirken, weil nach unserer Erfahrung der hepatische Progress häufig eine schnellere Dynamik zeigt als der pulmonale Metastasenprogress. In solchen Situationen kann im Einzelfall eine Leberresektion auch in Anbetracht einer beschränkten pulmonalen Metastasierung sinnvoll erscheinen. Bei Vorliegen einer disseminierten Peritonealkarzinose oder ausgedehnten retroperitonealen Metastasierung ist die Lebermetastasierung meist nicht prognoselimitierend - und von einer Leberresektion sollte in solchen Fällen meist Abstand genommen werden.

Neumann: Extrahepatische Tumormanifestationen sind ein negativer prognostischer Faktor für das Überleben nach Resektion kolorektaler Lebermetastasen (Ausnahme: Primärtumor und lokoregionäre Lymphknoten). Elias et al. [9] konnten jedoch bei gleichzeitiger Resektion von Lebermetastasen und extrahepatischen Metastasen ein tumorfreies 3- und 5-Jahres-Überleben von 22 und 11\% erreichen. Nach R0Resektion lebten nach 5 Jahren 29\% der Patienten. Gleiches gilt für resektable Lungenmetastasen. In einem Kollektiv von 26 hochselektiven Patienten berichteten Reddy et al. [10] über ein mittleres Überleben von 35 Monaten nach vollständiger Resektion von Lungen- und Lebermetastasen.

Bislang nicht eindeutig geklärt ist das zu empfehlende Vorgehen bei Lymphknotenmetastasen im Leberhilus. Diese gelten in vielen Zentren weiterhin als eine Kontraindikation für eine Leberresektion. Mehrere Studien belegen jedoch ein mögliches langfristiges Überleben. Jaeck et al. [11] haben zwei verschiedene Regionen von Lymphknotenmetastasen definiert, zum einen regionär, im Bereich des Leberhilus und retropankreatisch, zum anderen in der paraaortalen Region und am Truncus coeliacus. Bei Patienten mit regionalen Lymphknoten im Bereich des Leberhilus und retropankreatisch zeigte sich ein deutlich besseres 3-Jahres-Überleben. Patienten mit Lymphknotenmetastasen sind meines Erachtens gute Kandidaten für eine perioperative Chemotherapie.

Schlitt: Bei «Patienten mit ungünstiger Prognose» unter den oben genannten Aspekten ist eine operative Therapie im Ein- 
zelfall abzuwägen. Hierbei muss aber betont werden, dass der Nachweis eines extrahepatischen Tumors (Hiluslymphknoten, Lunge, lokal) nicht von vornherein als Palliativsituation angesehen werden darf. Auch in dieser Situation gilt: Wenn eine R0-Situation erreichbar ist, z.B. durch simultane oder sequenzielle Operation(en) oder sonstige destruierende Interventionen (z.B. Radiofrequenzablation (RFA) von Leberherden oder auch stereotaktische Bestrahlung von Lungenherden), sollte dies erwogen werden. Hier spielen bei der Entscheidung im Einzelfall - neben einer sehr umfangreichen Diagnostik - viele andere Faktoren eine Rolle: Begleiterkrankungen des Patienten, Alter, Tumorbiologie (z.B. ein sehr frühes Rezidiv oder Progress unter Chemotherapie). Häufig sind solche Entscheidungen auch nicht adäquat im Tumorboard zu treffen - ohne ganz genaue Kenntnis des einzelnen Patienten und intensiven Gesprächen mit dem Patienten und seinen Angehörigen.

Schulze-Bergkamen: Eine Leberresektion sollte prinzipiell nur dann erfolgen, wenn auch sämtliche extrahepatischen Tumormanifestationen R0-resezierbar sind. Bei R0-Resektabilität aller Manifestationen sollte eine Resektion angestrebt werden. Dabei sollte bei allen Patienten initial eine neoadjuvante Systemtherapie interdisziplinär diskutiert werden. Bei Vorliegen von sonstigen Fernmetastasen abgesehen von der Leber (lymphatisch, pulmonal) favorisieren wir zunächst die Einleitung einer systemischen Therapie, um Patienten mit ungünstiger Prognose zu identifizieren (weitere Metastasen bzw. Progress unter laufender systemischer Therapie; in diesem Fall kann die Leberresektion im individuellen Fall ihren onkologischen Stellenwert verlieren).

Frage 4: Was empfehlen Sie bei synchronen, operablen/ inoperablen Lebermetastasen eines kleinen, asymptomatischen Rektumkarzinoms?

Köhne: Die synchrone Metastasierung eines Rektumkarzinoms oder eines Kolonkarzinoms ist eine besondere Herausforderung. In der Regel sprechen der Lokaltumor und die Metastasen gleichwertig auf eine Chemotherapie an, d.h. bei einem nichtstenosierenden Tumor kann durchaus eine präoperative chemotherapeutische Behandlung sinnvoll sein, die zu einer Tumorkontrolle führt und damit ein Zeitfenster für eine Operation des Lokaltumors bzw. der Lebermetastasen schafft. Auch hier ist der individuelle Fall gefragt. Bei einem kleinen Rektumkarzinom und einer singulären Lebermetastase ist durchaus auch ein primäres operatives Vorgehen gefolgt von einer adjuvanten Chemotherapie sinnvoll. Bei einer Metastasierung, die überwiegend in der Leber ist, und an einem kleineren Rektumkarzinom würde ich auf jeden Fall eine Chemotherapie voranstellen, um eine Kontrolle der Haupttumorlast zu schaffen und erst eine Operation zu ermöglichen. Bei einem anderweitigen Vorgehen besteht die
Gefahr, dass peri- oder postoperativ bereits eine deutliche Progression der Lebermetastasen eintritt, die durch eine Verschlechterung des Allgemeinzustands oder eines Ikterus häufig die Chemotherapie verhindern kann.

Lang: Bei synchronen, resektablen Metastasen eines asymptomatischen Rektumkarzinoms kann im Sinne eines Liverfirst-Konzepts die Leberresektion an erster Stelle stehen, weil das Leben des Patienten in solchen Fällen eher durch die hepatische Metastasierung als durch den Primarius bedroht ist. Zudem ist mit einem intrahepatischen Tumorprogress unter Radio-/Chemotherapie des Rektumkarzinoms zu rechnen. Im Falle einer limitierten Leberresektion kann diese durchaus simultan mit der Rektumresektion erfolgen. Von einer Rektumresektion im Rahmen größerer Leberresektionen raten wir ab.

In diesen Fällen sollte nach kurativer Leberresektion die Therapie des Rektumkarzinoms stadiengerecht erfolgen. Im Stadium IV ist der Nutzen einer Radiochemotherapie für den Primärtumor zwar nicht hinreichend belegt, aber handelt es sich um eine limitierte hepatische Metastasierung bei lokal fortgeschrittenem Rektumkarzinom, erscheint eine primäre Leberresektion, gefolgt von einer Radio-/Chemotherapie mit sekundärer Rektumresektion zur lokalen Tumorkontrolle, sinnvoll.

Im Falle einer primär inoperablen Lebermetastasierung bei kleinem asymptomatischem Rektumkarzinom empfehlen wir primär eine systemische Chemotherapie mit kritischer Re-Evaluation der Operabilität der Lebermetastasen nach 3 und nach 6 Monaten.

Neumann: Die Abfolge von Chemotherapie und Leberresektion bei synchronen Lebermetastasen ist Gegenstand aktueller Diskussionen. Prospektiv randomisierte Vergleiche der unterschiedlichen Therapiestrategien fehlen. Die individuell häufig komplexen therapeutischen Strategiemöglichkeiten bei synchronen Lebermetastasen sollten obligat vor Einleitung jeglicher therapeutischer Maßnahmen in einem interdisziplinären Tumorboard besprochen werden.

Verschiedene Studien zeigten hinsichtlich der Morbidität und Mortalität gute Ergebnisse zum einzeitigen Vorgehen bei synchronen kolorektalen Lebermetastasen in einem ausgewählten Patientenkollektiv [12-14]. So ist in Abhängigkeit von der Komorbidität des Patienten und dem Ausmaß der notwendigen Leberresektion aus unserer Sicht eine einzeitige Resektion der Lebermetastasen und des Primarius gerechtfertigt. Retrospektive Daten zeigten hierbei eine Komplikationsrate von 16\% (Dindo-Clavien-Klassifikation 3 und 4) ohne signifikante Verzögerung der postoperativen Chemotherapie und ein dem zweizeitigen Vorgehen vergleichbares DFS und OS [15].

Bei operablen synchronen Lebermetastasen, K-RAS-Wildtyp und Erfüllung der weiteren Einschlusskriterien wird in unserer Klinik ein Einschluss in die PANTER-Studie ange- 
strebt. Ein zweizeitiges Vorgehen ist in dieser Studie ebenfalls möglich.

Die Wertigkeit des inversen Vorgehens («liver-first approach»), das eine Resektion der Lebermetastasen vor der Primärtumorresektion beinhaltet, ist noch nicht abschließend geklärt. Wir präferieren dieses Verfahren bei asymptomatischem Primarius und sehr hoher Tumorlast der Leber.

Für primär nichtoperable oder multiple Lebermetastasen und asymptomatischen Primarius im Rektum empfehlen wir eine frühzeitige systemische Chemotherapie und ein Restaging hinsichtlich einer möglichen sekundären Operabilität.

Schlitt: Bei definitiv inoperablen synchronen Lebermetastasen wird die Tumorlast in der Leber für den Patienten bezüglich der Prognose entscheidend sein - und nicht der Primärtumor. Daher ist in diesem Fall eine Operation des (asymptomatischen) Primärtumors nicht indiziert. Es sollte primär eine intensive systemische Therapie erfolgen, gegebenenfalls unter Hinzunahme eines Biologicals.

Bei potenziell resektablen synchronen Lebermetastasen muss abgewogen werden, ob primär eine chirurgische Therapie erfolgen soll oder ob eine neoadjuvante Behandlung sinnvoll erscheint. Im eigenen Vorgehen würde das in der Regel vom Ausmaß des Leberbefalls abhängig gemacht werden. Bei einer solitären Lebermetastase kann zunächst die Resektion der Lebermetastase erfolgen (Liver-first-Konzept), danach in einer zweiten Operation die Rektumresektion, gegebenenfalls nach lokaler Kurzzeitbestrahlung. Bei mehreren oder grenzwertig resektablen Lebermetastasen sollte zunächst eine intensive Chemotherapie (gegebenenfalls mit Biological) erfolgen, danach die Leberresektion und im letzten Schritt die Rektumresektion, gegebenenfalls nach Kurzzeitbestrahlung. Grundsätzlich erscheint in der Situation von synchronen Lebermetastasen beim Rektumkarzinom eine «klassische» Langzeit-Radiochemotherapie aufgrund der langen Therapiedauer mit (für die Situation) vermutlich zu schwacher systemisch wirksamer Therapie nicht sinnvoll, sodass das Risiko einer deutlichen Progredienz der Lebermetastasen besteht.

Schulze-Bergkamen: Operable Lebermetastasen: Ein- oder zweizeitiges Vorgehen nach Maßgabe des Abdominalchirurgen; interdisziplinäre Diskussion einer perioperativen Chemotherapie; bei zweizeitigem Vorgehen Reihenfolge der Operationen nach Maßgabe des Abdominalchirurgs.

Potenziell operable Lebermetastasen: Zunächst remissionsinduzierende Systemtherapie.

Irresektable Lebermetastasen: Ein asymptomatischer Primärtumor sollte nicht reseziert werden (hier sei allerdings auf die laufende Multicenterstudie «Synchronous» hingewiesen); Einleitung systemische Therapie; gegebenenfalls Strahlentherapie zur lokalen Kontrolle des Rektumkarzinoms (Stellungnahme Radioonkologe).

\section{Frage 5: Was soll nach Downstaging durch} Chemotherapie mit nicht mehr sichtbaren Metastasen passieren, insbesondere wenn diese zuvor beidseits multipel waren?

Köhne: Etwa 15\% der klinisch kompletten Remissionen bei Lebermetastasen sind auch pathologisch komplette Remissionen. Handelt es sich um eine begrenzte Anzahl von Metastasen, sollte der Versuch unternommen werden, diese in ihren ehemaligen Grenzen mitzuresezieren. Bei primär nichtoperablen Lebermetasten wird durch eine Chemotherapie eine Tumorverkleinerung erreicht, mit zum Teil komplettem Verschwinden der vorher sichtbaren Metastasen. In diesem Fall erfolgt eine Resektion der noch sichtbaren Metastasen - in der Hoffnung, dass einige andere Metastasen dauerhaft von der Chemotherapie zerstört werden. Dies ist letztlich das Wesen einer neoadjuvanten Chemotherapie bei primär nichtresektablen Metastasen; zumindest in der Gruppe von Patienten, die aufgrund von multiplen Metastasen nicht operabel sind. Sind alle Metastasen nicht mehr sichtbar, kann gewartet werden, bis die Metastasen wieder auftauchen, um sie dann zu operieren. Dies verlangt engmaschige Untersuchungen.

Lang: Bei sogenannten «verschwundenen» Lebermetastasen, die sich nach Chemotherapie weder radiologisch noch intraoperativ wiederfinden lassen, kann in ca. 50\% der Fälle mit einer «echten» Vollremission gerechnet werden. Die Datenlage zum chirurgischen Vorgehen in solchen Situationen ist nicht eindeutig. Wurde die Chemotherapie im Sinne der EORTC-Studie bei primär resektablen Metastasen in neoadjuvanter Intention durchgeführt, kann eine Resektion entlang der initialen Tumorausdehnung - bei fehlenden belastbaren Daten - durchaus gerechtfertigt werden. In vielen Fällen dürfte aber die Chemotherapie bei initial nicht oder nicht optimal resektablen Metastasen, eventuell bei bilobärer Metastasierung, durchgeführt worden sein. In diesen Fällen ist eine Resektion gemäß der initialen Tumorausdehnung per Definition nicht möglich, und die Resektion sollte sich am aktuellen, posttherapeutischen Tumorausmaß orientieren. In diesen Fällen sollte eine Kernspintomographie der Leber erfolgen. Eine Resektion sollte sich auf nachweisbare Befunde mit parenchymsparenden Resektionen beschränken, da dieses Vorgehen im Falle eines Rezidivs eventuell eine weitere Leberresektion ermöglicht.

Neumann: Die Beobachtung, dass eine präoperative Chemotherapie zu einer radiologischen kompletten Remission führen kann, stellt nach wie vor eine Herausforderung für die weitere Therapieplanung dar. Problematisch ist insbesondere die Beurteilung der kompletten klinischen und pathologischen Remission angesichts einer zum Teil widersprüchlichen Datenlage. In verschiedenen Studien [16, 17] zeigten sich in ca. $30-80 \%$ Residualtumore bzw. Frührezidive trotz prä- 
operativ computertomographisch nachgewiesener kompletter Remission. In einer anderen Untersuchung an über 400 Patienten waren nach Chemotherapie bei 9\% der Patienten keine Lebermetastasen mehr nachweisbar [18]. 44 von 68 resezierten Patienten zeigten anschließend eine komplette pathologische Remission, und 31 von 50 nichtoperierten Patienten hatten nach einem Jahr eine persistierende radiologische/ klinische komplette Remission [18].

Problematisch ist die Tatsache, dass derzeit keine verlässlichen prognostischen Marker zur Beurteilung einer kompletten Remission zur Verfügung stehen. Adam et al. [19] beobachteten in einer Studie mit über 700 Patienten nach präoperativer Chemotherapie eine komplette pathologische Remission bei $4 \%$ der Patienten. Hierbei waren ein Lebensalter unter 60 Jahren, niedrige CEA-Spiegel und eine Metastasengröße unter $3 \mathrm{~cm}$ unabhängige prädiktive Faktoren für eine komplette pathologische Remission.

Die radiologische Beurteilung einer kompletten Remission sollte grundsätzlich mittels CT und Kernspintomographie geprüft werden [20]. Aus unserer Sicht ist insbesondere bei sowohl im CT als auch im MRT nicht mehr nachweisbaren beidseits multiplen Metastasen derzeit ein nichtchirurgisches Vorgehen bzw. eine engmaschige Nachsorge gerechtfertigt. Dagegen empfehlen wir bei begrenzter Tumorlast eine weitere Evaluation mittels Laparoskopie und IOUS und bei sonographischem Tumornachweis die Metastasenresektion.

Schlitt: Falls die Gesamtkonstellation einen kurativen Ansatz erlaubt, sollte versucht werden, alle primären Herde intraoperativ - so weit möglich - zu lokalisieren und zu resezieren oder zu abladieren. Hierzu müssen zum einen die CT- bzw. MRT-Bilder aus der Zeit vor der Therapie vorhanden sein und in die OP-Planung mit einbezogen werden. Zusätzlich kann die intraoperative KM-Sonographie (CE-IOUS) auch die Lokalisation sehr kleiner «Rest-Herde» intraoperativ möglich machen, auch wenn diese präoperativ im CT oder MRT nicht sichtbar waren. Wenn eine Lokalisation auch intraoperativ gar nicht möglich ist und eine «blinde» Resektion oder Ablation zu riskant erscheint (z.B. im Zusammenhang mit weiteren Resektionen), muss der Befund belassen werden. Gegebenenfalls kann dann zweizeitig eine erneute Resektion oder Ablation erfolgen.

Schulze-Bergkamen: Nur bei einem geringen Anteil von nicht mehr sichtbaren Metastasen liegt auch eine pathologische Komplettremission vor [19]. Deshalb sollten auch bei nicht mehr sichtbaren Metastasen in der Bildgebung ein leberchirurgischer Eingriff erfolgen und auch die Areale der nicht mehr sichtbaren Metastasen reseziert werden (gegebenenfalls Einbeziehung IOUS und Gewebeprobe/Schnellschnitt; Stellungnahme Abdominalchirurg erforderlich).
Frage 6: Welchen Stellenwert haben für Sie lokal ablative Verfahren (RFA, Afterloading, SIRT, selektive Embolisierung usw.)?

Köhne: Lokal ablative Verfahren können eine sinnvolle Ergänzung zu einer Chemotherapie sein. Im Rahmen einer EORTC-Studie wurde die alleinige chemotherapeutische Behandlung mit einer chemotherapeutischen Behandlung plus RFA verglichen. In diesem Fall ergab sich eine Verbesserung des DFS zugunsten des lokal ablativen Verfahrens. Ein lokal destruierendes Verfahren im Sinne einer RFA kann eine Operation nicht ersetzen, die immer noch als Goldstandard der Therapiebehandlung gilt. Allerdings kann unter palliativen Gesichtspunkten ein solches Verfahren durchaus sinnvoll sein und muss im Einzelfall interdisziplinär diskutiert werden.

Lang: Diese Verfahren haben ihren Stellenwert bei nichtresektablen Lebermetastasen. Die RFA kann zudem ergänzend im Rahmen einer Leberresektion durchgeführt werden, wenn eine komplette Resektion einzelner Metastasen mit einem $\mathrm{zu}$ hohen Risiko eines Leberversagens verbunden wäre. Die selektive interne Radiotherapie (SIRT) als Radioembolisation der Leber bekommt zunehmende Bedeutung in der Therapie chemoresistenter, irresektabler Lebermetastasen. Als Alternative in der First-line-Therapie ist die SIRT jedoch noch nicht anzusehen, da es keine prospektiven Daten $\mathrm{zu}$ dieser Therapieform gibt. Insgesamt kommen diese Verfahren bei Lebermetastasen deutlich seltener zur Anwendung als bei primären Leberzellkarzinomen.

Neumann: Das am besten untersuchte ablative Verfahren ist die RFA, jedoch auch entsprechend aktueller Übersichtsarbeiten mit nicht eindeutiger Studienlage [21]. In einer multizentrischen Studie von Lencioni et al. [22] an 423 mittels RFA behandelten Patienten mit insgesamt 615 metachronen kolorektalen Läsionen (mittlere Tumorgröße 2,7 cm) zeigte sich ein lokaler Tumorprogress bei $25 \%$ der Patienten. Die 1-, 3- und 5-Jahres-Überlebensraten betrugen 86, 47 und 24\%. Dies entspricht den Daten von Abdalla et al. [23], die gezeigt haben, dass eine lokale Tumorprogression nach RFA wahrscheinlicher ist als nach Resektion. Die Lokalrezidivrate ist dabei abhängig von der Lage und Größe der Tumoren und variiert zwischen 10 und 47\%. Die 3-Jahres-Überlebenswahrscheinlichkeit liegt zwischen 20 und $36 \%$ und ist schwer einzuschätzen, da in den Studien meist Patienten mit ungünstiger Prognose behandelt wurden. Bei größeren bzw. gefäßnahen Tumoren stellt die interstitielle Brachytherapie in Afterloading-Technik ein Alternativverfahren mit guten Kontrollraten dar [24]. Einen wichtigen Stellenwert hat in diesem Zusammenhang auch die lokale Chemotherapie. In einer aktuellen Untersuchung zu initial nichtresektablen Lebermetastasen führte sie zu einer hohen Rate an Vollremissionen [25].

Da es bisher keine prospektiven Daten zu den lokalen Ablationsverfahren im Vergleich zu einer kurativen Leberresek- 
tion gibt, können diese Verfahren nicht als Alternative zu einer Leberresektion empfohlen werden. Sie haben jedoch einen wichtigen Stellenwert als ergänzendes Instrument, um bei sonst nicht R0-resektablen Patienten eine Tumordestruktion $\mathrm{zu}$ erreichen. Dies gilt insbesondere für Patienten mit hoher Komorbidität, bei ungünstiger anatomischer Metastasenlokalisation, bei eingeschränkter Leberfunktion oder bei gleichzeitiger extrahepatischer Tumormanifestation.

Schlitt: Ablative Verfahren wie die RFA haben einen relevanten Stellenwert, vor allem bei kleinen, ungünstig gelegenen Rezidiv-Lebermetastasen und - in Kombination mit der Chirurgie - bei multiplen Metastasen. Damit können diese Verfahren zum Erreichen einer R0-Situation beitragen. Die Ergebnisse der ablativen Verfahren hängen jedoch sehr von der Erfahrung des Durchführenden ab. Sie sollten daher auf Zentren beschränkt werden und immer eine gemeinsame Besprechung in einem interdisziplinären Tumorboard voraussetzen. Ob neuere Verfahren wie die Elektroporation (die auch in der Nähe von Gefäßen und Gallengängen vermutlich effizienter und sicherer als die RFA durchführt werden kann) weitere Fortschritte bringen, muss die Zukunft zeigen.

Die SIRT ist ein interessantes Therapieverfahren, das bei irresektablen Lebermetastasen erwogen werden kann. Dies gilt insbesondere, wenn es zu einem Tumorprogress unter Chemotherapie gekommen ist bzw. die systemischen chemotherapeutischen Optionen «ausgereizt» sind und der Tumorbefall der Leber prognostisch führend ist.

Ein weiteres, im Einzelfall anwendbares Therapieverfahren ist die stereotaktische Radiotherapie, die allerdings weniger in der Leber, sondern eher bei Lungenmetastasen zum Einsatz kommt.

Die eigene Arbeitsgruppe hat keine Erfahrung mit Afterloading und selektiver Embolisierung von kolorektalen Lebermetastasen.

Schulze-Bergkamen: RFA: Kann die Möglichkeiten eines kurativen leberchirurgischen Eingriffs erweitern (Stellungnahme Abdominalchirug erforderlich); im palliativen Setting in Einzelfällen bei Patienten mit singulären Lebermetastasen (z.B. bei fehlenden Optionen für systemische Therapieansätze oder fehlender Option für eine Leberchirurgie bei eingeschränkter Narkosefähigkeit/Multimorbidität) (Stellungnahme interventioneller Radiologe).

SIRT: In palliativer Situation bei ausschließlich oder überwiegend auf die Leber beschränkter Metastasierung und Versagen einer systemischen Therapie.

Selektive Embolisierung: Stellungnahme Radiologe erforderlich; am eigenen Zentrum ohne wesentlichen Stellenwert.

Afterloading: Stellungnahme Radioonkologe erforderlich, am eigenen Zentrum ohne wesentlichen Stellenwert.

\section{Teilnehmer}

Prof. Dr. Claus-Henning Köhne

Klinik für Onkologie und Hämatologie

Klinikum Oldenburg gGmbH

Rahel-Straus-Straße 10, 26133 Oldenburg, Deutschland

onkologie@klinikum-oldenburg.de

Prof. Dr. med. Hauke Lang

Klinik und Poliklinik für Allgemein- und Abdominalchirurgie

Johannes Gutenberg-Universität Mainz

Langenbeckstraße 1, 55131 Mainz, Deutschland

Lang@ach.klinik.uni-mainz.de

Univ.-Prof. Dr. med. Ulf Peter Neumann

Klinik für Allgemein-, Viszeral- und Transplantationschirurgie

Universitätsklinikum Aachen

Pauwelsstraße 30, 52074 Aachen, Deutschland

chirurgie@ukaachen.de

\section{Prof. Dr. Hans J. Schlitt}

Klinik und Poliklinik für Chirurgie

Universitätsklinikum Regensburg (UKR)

Franz-Josef-Strauß-Allee 11, 93053 Regensburg, Deutschland

hans.schlitt@klinik.uni-regensburg.de

Prof. Dr. Henning Schulze-Bergkamen

Abteilung für Medizinische Onkologie

Nationales Centrum für Tumorerkrankungen (NCT)

Universitätsklinikum Heidelberg

Im Neuenheimer Feld 460, 69120 Heidelberg, Deutschland

henning.schulze@med.uni-heidelberg.de

\section{Literatur}

1 Nordlinger B, Sorbye H, Glimelius B, Poston GJ, Schlag PM, Rougier P, Bechstein WO, Primrose JN, Walpole ET, Finch-Jones M, Jaeck D, Mirza D, Parks RW, Collette L, Praet M, Bethe U, Van Cutsem E, Scheithauer W, Gruenberger T; EORTC Gastro-Intestinal Tract Cancer Group; Cancer Research UK; Arbeitsgruppe Lebermetastasen und -tumoren in der Chirurgischen Arbeitsgemeinschaft Onkologie (ALM-CAO); Australasian Gastro-Intestinal Trials Group (AGITG); Fédération Francophone de Cancérologie Digestive (FFCD): Perioperative chemotherapy with FOLFOX4 and surgery versus surgery alone for resectable liver metastases from colorectal cancer (EORTC Intergroup trial 40983): a randomised controlled trial. Lancet 2008;371:1007-1016.

2 Mitry E, Fields AL, Bleiberg H, Labianca R, Portier G, Tu D, Nitti D, Torri V, Elias D, O'Callaghan C, Langer B, Martignoni G, Bouche O, Lazorthes F, Van CE, Bedenne L, Moore MJ, Rougier P: Adjuvant chemotherapy after potentially curative resection of metastases from colorectal cancer: a pooled analysis of two randomized trials. J Clin Oncol 2008;26:4906-4911.
3 Reddy SK, Zorzi D, Lum YW, Barbas AS, Pawlik TM, Ribero D, Abdalla EK, Choti MA, Kemp C, Vauthey JN, Morse MA, White RR, Clary BM: Timing of multimodality therapy for resectable synchronous colorectal liver metastases: a retrospective multi-institutional analysis. Ann Surg Oncol 2009;16:1809-1819.

4 Adam R, Bhangui P, Poston G, Mirza D, Nuzzo G, Barroso E, Ijzermans J, Hubert C, Ruers T, Capussotti L, Ouellet JF, Laurent C, Cugat E, Colombo $\mathrm{PE}$, Milicevic M: Is perioperative chemotherapy useful for solitary, metachronous, colorectal liver metastases? Ann Surg 2010;252:774-787. 
5 Khan AZ, Morris-Stiff G, Makuuchi M: Patterns of chemotherapy-induced hepatic injury and their implications for patients undergoing liver resection for colorectal liver metastases. J Hepatobiliary Pancreat Surg 2009;16:137-144.

6 Kishi Y, Zorzi D, Contreras CM, Maru DM, Kopetz S, Ribero D, Motta M, Ravarino N, Risio M, Curley SA, Abdalla EK, Capussotti L, Vauthey JN: Extended preoperative chemotherapy does not improve pathologic response and increases postoperative liver insufficiency after hepatic resection for colorectal liver metastases. Ann Surg Oncol 2010; 17:2870-2876

7 Nordlinger B, Vauthey JN, Poston G, Benoist S, Rougier P, Van Cutsem E: The timing of chemotherapy and surgery for the treatment of colorecta liver metastases. Clin Colorectal Cancer 2010;9: 212-218.

8 Rubbia-Brandt L, Lauwers GY, Wang H, Majno PE, Tanabe K, Zhu AX, Brezault C, Soubrane O, Abdalla EK, Vauthey JN, Mentha G, Terris B: Sinusoidal obstruction syndrome and nodular regenerative hyperplasia are frequent oxaliplatin-associated liver lesions and partially prevented by bevacizumab in patients with hepatic colorectal metastasis. Histopathology 2010;56:430-439.

$\checkmark$ Elias D, Liberale G, Vernerey D, Pocard M, Ducreux M, Boige V, Malka D, Pignon JP, Lasser P: Hepatic and extrahepatic colorectal metastases: when resectable, their localization does not matter, but their total number has a prognostic effect. Ann Surg Oncol 2005;12:900-909.

10 Reddy RH, Kumar B, Shah R, Mirsadraee S, Papagiannopoulos K, Lodge P, Thorpe JA: Staged pulmonary and hepatic metastasectomy in colorectal cancer - is it worth it? Eur J Cardiothorac Surg 2004;25:151-154.

-11 Jaeck D, Bachellier P, Guiguet M, Boudjema K, Vaillant JC, Balladur P, Nordlinger B: Long-term survival following resection of colorectal hepatic metastases. Association Francaise de Chirurgie. Br J Surg 1997;84:977-980.
12 Chua HK, Sondenaa K, Tsiotos GG, Larson DR, Wolff BG, Nagorney DM: Concurrent vs. staged colectomy and hepatectomy for primary colorectal cancer with synchronous hepatic metastases. Dis Colon Rectum 2004;47:1310-1316.

13 Hillingso JG, Wille-Jorgensen P: Staged or simultaneous resection of synchronous liver metastases from colorectal cancer - a systematic review. Colorectal Dis 2009;11:3-10.

14 Martin R, Paty P, Fong Y, Grace A, Cohen A, DeMatteo R, Jarnagin W, Blumgart L: Simultaneous liver and colorectal resections are safe for synchronous colorectal liver metastasis. J Am Coll Surg 2003;197:233-241.

15 Boostrom SY, Vassiliki LT, Nagorney DM, Wolff BG, Chua HK, Harmsen S, Larson DW: Synchronous rectal and hepatic resection of rectal metastatic disease. J Gastrointest Surg 2011;15:15831588.

16 Benoist S, Brouquet A, Penna C, Julie C, El-Hajjam M, Chagnon S, Mitry E, Rougier P, Nordlinger B: Complete response of colorectal liver metastases after chemotherapy: does it mean cure? J Clin Oncol 2006;24:3939-3945.

17 Tanaka K, Takakura H, Takeda K, Matsuo K, Nagano Y, Endo I: Importance of complete pathologic response to prehepatectomy chemotherapy in treating colorectal cancer metastases. Ann Surg 2009;250:935-942.

18 Auer RC, White RR, Kemeny NE, Schwartz LH, Shia J, Blumgart LH, DeMatteo RP, Fong Y, Jarnagin WR, D'Angelica MI: Predictors of a true complete response among disappearing liver metastases from colorectal cancer after chemotherapy. Cancer 2010;116:1502-1509.

9 Adam R, Wicherts DA, de Haas RJ, Aloia T, Levi F, Paule B, Guettier C, Kunstlinger F, Delvart V, Azoulay D, Castaing D: Complete pathologic response after preoperative chemotherapy for colorectal liver metastases: myth or reality? J Clin Oncol 2008;26:1635-1641.
0 Gaujoux S, Goere D, Dumont F, Souadka A, Dromain C, Ducreux M, Elias D: Complete radiological response of colorectal liver metastases after chemotherapy: what can we expect? Dig Surg 2011; 28:114-120.

1 Wong SL, Mangu PB, Choti MA, Crocenzi TS, Dodd GD, III, Dorfman GS, Eng C, Fong Y, Giusti AF, Lu D, Marsland TA, Michelson R, Poston GJ, Schrag D, Seidenfeld J, Benson AB 3rd: American Society of Clinical Oncology 2009 clinical evidence review on radiofrequency ablation of hepatic metastases from colorectal cancer. J Clin Oncol 2010;28:493-508.

22 Lencioni R, Crocetti L, Cioni D, Della PC, Bartolozzi C: Percutaneous radiofrequency ablation of hepatic colorectal metastases: technique, indications, results, and new promises. Invest Radiol 2004;39:689-697.

23 Abdalla EK, Vauthey JN, Ellis LM, Ellis V, Pollock R, Broglio KR, Hess K, Curley SA: Recurrence and outcomes following hepatic resection, radiofrequency ablation, and combined resection/ ablation for colorectal liver metastases. Ann Surg 2004;239:818-825.

4 Ricke J, Mohnike K, Pech M, Seidensticker M, Ruhl R, Wieners G, Gaffke G, Kropf S, Felix R, Wust P: Local response and impact on survival after local ablation of liver metastases from colorectal carcinoma by computed tomography-guided high-dose-rate brachytherapy. Int J Radiat Oncol Biol Phys 2010;78:479-485.

25 Goere D, Gaujoux S, Deschamp F, Dumont F, Souadka A, Dromain C, Ducreux M, Elias D: Patients operated on for initially unresectable colorectal liver metastases with missing metastases experience a favorable long-term outcome. Ann Surg 2011;254:114-118. 